FORMATION Formation emploi

Revue française de sciences sociales

99 | juillet-septembre 2007

Les usages sociaux de la compétence

\title{
Dominique Lhuilier, Le travail comme épreuve et lieu de construction du sujet
}

(2006), Cliniques du travail, Paris, Érès.

\section{Pierre Roche}

\section{OpenEdition}

\section{Journals}

Édition électronique

URL : http://journals.openedition.org/formationemploi/1524

DOI : 10.4000/formationemploi.1524

ISSN : 2107-0946

\section{Éditeur}

La Documentation française

Édition imprimée

Date de publication : 1 juillet 2007

Pagination : 139-141

ISSN : 0759-6340

\section{Référence électronique}

Pierre Roche, "Dominique Lhuilier, Le travail comme épreuve et lieu de construction du sujet », Formation emploi [En ligne], 99 | juillet-septembre 2007, mis en ligne le 19 février 2009, consulté le 30 octobre 2020. URL : http://journals.openedition.org/formationemploi/1524 ; DOI : https://doi.org/ 10.4000/formationemploi.1524 


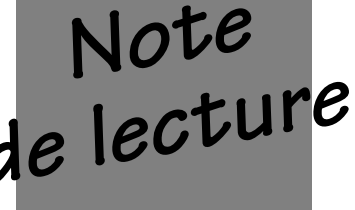

\section{Le travail comme épreuve et lieu de construction du sujet}

\author{
Par Pierre Roche*
}

Dominique Lhuilier

\section{Cliniques du travail}

érès
Voici un ouvrage que le lecteur aurait tort de seulement parcourir, qui mérite d'être lu et relu, dont le propos doit être confronté à sa propre expérience; à ce qu'il vit ou à ce dont il est témoin au quotidien sur la scène du travail. Car à cela il donne sens. Un ouvrage qui nous parle d'une condition humaine faite de souffrance plus que de plaisir, d'un malaise individuel et/ou collectif qui, comme en témoigne la montée des préoccupations et des

demandes sociales, ne cesse de grandir, en lien avec les transformations productives et les nouvelles formes du rapport subjectif au travail. Son propos est théorique mais point difficile d'accès car toujours fortement appuyé sur des exemples précis de situations de travail (dans les usines, les centrales nucléaires, le milieu carcéral, l'hôpital, la police...) qui, chaque fois, ont fait l'objet d'une analyse concrète.

Que l'on ne se méprenne pas cependant sur le dessein poursuivi par Dominique Lhuilier au travers de cet ouvrage : elle ne s'inscrit pas dans la filiation de Louis René Villermé en nous dressant le tableau noir de l'état physique et moral des travailleurs de ce début du XXI ${ }^{\mathrm{e}}$ siècle afin d'alerter les pouvoirs publics. Son objectif est autre ; il est tout d'abord de nous faire accéder à une meilleure compréhension du champ des cliniques du travail, lequel peut, de prime abord, en raison des multiples approches qui le traversent et le structurent, apparaître plus éclaté qu'unifié. Contentons-nous de citer en guise de repères quelques noms comme Louis Le Guillant pour la psychopathologie du travail ; Gérard Mendel pour la socio-analyse; Christophe Dejours pour la psychodynamique du travail ou encore Yves Clot pour la clinique de l'activité. Au terme de la lecture de son ouvrage, on voit mieux en effet sur quoi les chemins des uns et des autres convergent, et sur quoi

Pierre Roche est sociologue au Centre d'études et de recherche sur les qualifications (Céreq). II est président du Comité de sociologie clinique de l'Association française de sociologie et membre du Comité de rédaction de la Nouvelle Revue de Psychosociologie. Ses recherches portent tout particulièrement sur l'analyse du travail et les processus de professionnalisation. Il est notamment l'auteur de Sociologie clinique; enjeux théoriques et méthodologiques, avec Vincent de Gaulejac et Fabienne Hanique, Erès, 2007 ; La proximité à l'épreuve de l'économie de la débrouille, Mission Sida Toxicomanie de la Ville de Marseille, Céreq, 2006 ; Précarisation du travail et lien social. Des hommes en trop, avec Frédéric Abécassis, Paris, L'Harmattan, 2001. 
ils divergent. Et les choix théoriques et méthodologiques de Dominique Lhuilier, aussi, s'éclairent par leur confrontation à ceux de ces auteurs. Son objectif est ensuite de nous faire entendre que la clinique, quelle que soit l'approche adoptée, est avant tout une pratique et que ce n'est point seulement son domaine d'objet qui la spécifie comme telle, autrement dit le fait de s'intéresser au vécu singulier, à la souffrance au travail ou encore à la dimension affective des relations sociales. L'auteure s'attache alors à nous présenter les traits les plus saillants de cette pratique : construction d'un espace où les salariés peuvent surmonter leurs difficultés à dire le travail, les défenses qu'ils y déploient mais aussi l'intelligence de la pratique qu'ils mobilisent lorsqu'ils sont confrontés à des situations inédites, changeantes, imprévues; coopération étroite entre chercheurs et acteurs ; coconstruction du sens de l'expérience et de la situation; retour réflexif du chercheur sur son implication et sur les effets de sa rencontre avec les acteurs du point de vue de la dynamique même de l'enquête; visée compréhensive, analytique mais aussi transformatrice vis-à-vis des situations de travail qui sont à l'origine de ce malaise... Une pratique qui, on le voit, bouleverse la figure du chercheur. Celui-ci, pour le coup, n'est plus cet expert qui disposerait du savoir dont l'acteur manquerait et qui mettrait les résultats de sa recherche au service de ce dernier, qui n'aurait alors plus qu'à transformer ses recommandations-préconisations en décisionsactions. Il est un intervenant qui, au même titre que les autres acteurs, mais à partir de sa place spécifique, tente de comprendre en agissant et d'agir en comprenant, adoptant ici une posture bien connue de ceux qui ont pris le parti de la recherche-action.

Mais qu'est-ce donc que le travail ? Ou plutôt, que devient-il une fois situé dans cette perspective clinique? Selon nous, une des originalités de l'auteure est sans doute de promouvoir une vision large du travail. D'abord, en le sortant de ses seules formes instituées, et notamment de la seule sphère de l'emploi, y intégrant les activités à l'œuvre dans le monde domestique, le bénévolat, le syndicalisme, la politique ou encore la débrouillardise sociale propre à ceux qui n'ont pas d'emploi ou qui ont un emploi ne leur permettant pas de vivre décemment. Ceci, à bien y réfléchir, est finalement peu courant et résonne comme un appel à lancer des recherches empiriques sur ces formes du travail quelque peu délaissées par la communauté scientifique. Ensuite, en insistant sur son caractère fondamentalement social. L'auteure nous conduit au plus loin de toute vision solipsiste d'un travail qui ne mettrait finalement en présence qu'un homme et une tâche puisque celui-ci est avant tout: "une scène où se jouent simultanément et dialectiquement le rapport à soi, le rapport à autrui et le rapport au réel.» Enfin, en se démarquant de toute conception qui ne verrait dans le travail que contraintes, aliénation et souffrance. L'auteure, d'ailleurs, ne se contente pas d'évoquer son lien avec la liberté, le plaisir, voire la sublimation mais porte au jour ses fonctions tout à la fois sociales (au travers de la production du lien social) et psychologiques (au travers de la séparation d'avec soi-même). Précisément parce que le travail est une épreuve psychique, il est aussi le lieu où le sujet peut se construire.

Avant de clore ce compte-rendu, il nous faut encore dire quelques mots des deux derniers chapitres de cet ouvrage, consacrés à des concepts qui sont devenus aujourd'hui, à certains égards, incontournables dans le champ de la clinique, ceux de stratégie défensive (Dejours C., Travail et usure mentale, Paris, Bayard, 2000) et d'activité empêchée (Clot Y., La fonction psychologique du travail, Paris, PUF, 1999) car l'un et l'autre gagnent en densité à l'issue d'une discussion passionnante parce que tout à la fois théorique et bien étayée sur des cas concrets. Nous semble particulièrement fécond, par exemple, le fait de ne plus se contenter de référer l'élaboration de la stratégie défensive au seul collectif de travail en place dans tel ou tel lieu. Ainsi, la profession ou la communauté de métier en tant que telle y participe pleinement parce qu'elle offre à chacun de ses membres de nombreux repères identificatoires. L'ensemble social, aussi, en ce qu'il assigne une place imaginaire à cette profession ou communauté de métier dans l'ordre du monde qui, grâce à cela, n'est plus un «chaos indifférencié », pour reprendre l'expression de Cornelius Castoriadis dans L'institution imaginaire de la société (Paris, Le Seuil, 1975). Le concept d'activité empêchée, proposé par Yves Clot mais dont la quête de filiation nous ferait remonter sans doute jusqu'à la problématique spinoziste de la puissance d'agir, nous engage dans un champ de 
recherche encore très largement en friche dans la mesure où l'on avait tendance jusqu'ici à rabattre toute l'activité réelle sur la seule activité réalisée. Pourtant, pour ne retenir qu'une dimension de ce champ, nombre de salariés, aujourd'hui, sont en souffrance parce qu'une grande part de leur activité est en souffrance (en attente de réalisation). On comprend alors que les questions posées dans l'introduction concernant notamment les diverses formes $\mathrm{du}$ malaise au travail ne trouveront peut-être de réponses satisfaisantes qu'à condition de développer aussi des recherches sur ce versant de l'activité. $\mathrm{Au}$ total, nous ne saurions que conseiller vivement cet ouvrage à tous ceux qui sont désireux de mieux cerner les enjeux sociaux et subjectifs qui se nouent autour du travail et les questions théoriques qui s'y rattachent. Parions enfin qu'il incitera à développer de nouvelles recherches cliniques, tout particulièrement sur des dimensions que sa lecture, précisément, permet de sortir de l'ombre.

Référence de l'ouvrage :

Lhuilier D. (2006), Cliniques du travail, Paris, Érès. 


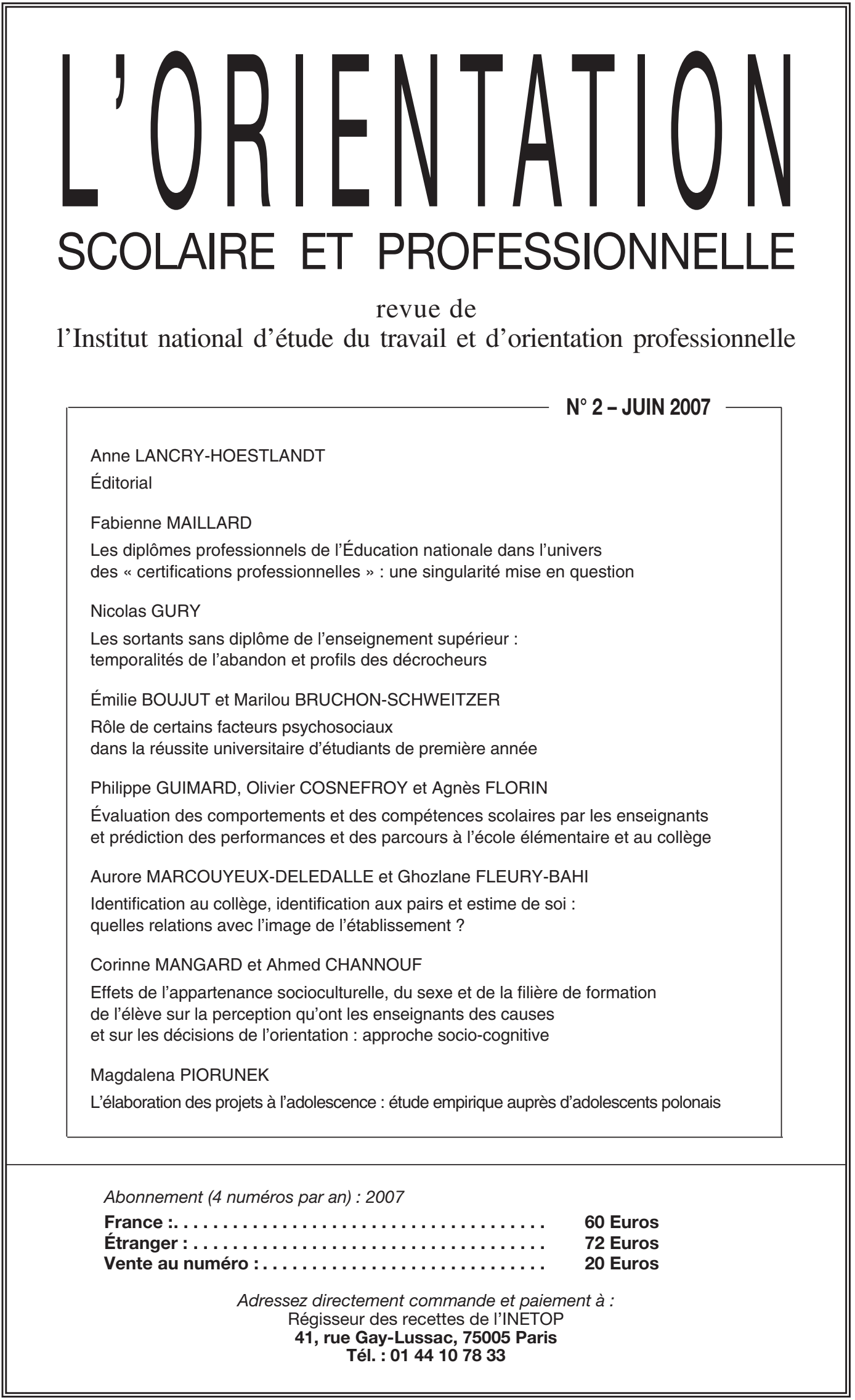

